\title{
Observation of K-Shell Soft X Ray Emission of Mitrogen Irradiated by XUV-Free Electron Laser FLASH at Intensities Greater Than $10^{16} \mathbf{W} / \mathbf{c m}^{2}$
}

\author{
E. Galtier ${ }^{1}$, F.B. Rosmej*1, O. Renner ${ }^{2}$, L. Juha ${ }^{2}$, J. Chalupsky ${ }^{2}$, J.-C. Gauthier ${ }^{3}$, S. White ${ }^{4}$, \\ D. Riley ${ }^{4}$, S. Vinko ${ }^{5}$, T. Witcher ${ }^{5}$, J. Wark ${ }^{5}$, B. Nagler ${ }^{6}$, R.W. Lee ${ }^{7}$, A.J. Nelson ${ }^{7}$, and S. Toleikis ${ }^{8}$ \\ ${ }^{1}$ Université Pierre et Marie Curie, UMR 7605, LULI, case 128, 4 place Jussieu, 75252 Paris Cedex 05, France \\ 2 Institute of Physics ASCR, Prague, Czech Republic \\ ${ }^{3}$ Centre Lasers Intenses et Applications CELIA, Bordeaux, France \\ ${ }^{4}$ Queens University of Belfast, UK \\ ${ }^{5}$ Department of Physics, Clarendon Laboratory, University of Oxford, UK \\ ${ }^{6}$ SLAC National Accelerator Laboratory, California, USA \\ ${ }^{7}$ Lawrence Livermore National Laboratory, California, USA \\ ${ }^{8}$ Deutsches Elektronen-Synchrotron DESY, Hamburg, Germany
}

Received 25 July 2010, accepted 25 August 2010

Published online 16 March 2011

Key words Soft X-ray diagnostic, Free Electron Laser, THM crystal.

\begin{abstract}
In the past few years, the development of light sources of the $4^{\text {th }}$ generation, namely XUV/X-ray Free Electron Lasers provides to the scientific community outstanding tools to investigate matter under extreme conditions never obtained in laboratories so far. As theory is at its infancy, the analysis of matter via the self-emission of the target is of central importance. The characterization of such dense matter is possible if photons can escape the medium. As the absorption of K-shell X-ray transitions is minimal, it plays a key role in this study. We report here the first successful observation of K-shell emission of nitrogen at $0.43 \mathrm{keV}$ using an XUV-Free Electron Laser to irradiate solid Boron Nitride targets under exceptional conditions: photon energy of $92 \mathrm{eV}$, pulse duration of $\sim 20 \mathrm{fs}$, micro focusing leading to intensities larger than $10^{16} \mathrm{~W} / \mathrm{cm}^{2}$. Using a Bragg crystal of THM coupled to a CCD, we resolved K-shell line emission from different charge states. We demonstrate that the spectroscopic data allow characterization of electron heating processes when X-ray radiation is interacting with solid matter. As energy transport is non-trivial because the light source is monochromatic, these results have an important impact on the theory.
\end{abstract}

(c) 2011 WILEY-VCH Verlag GmbH \& Co. KGaA, Weinheim

\section{Introduction}

Investigation of matter under extreme conditions is of great challenge for a large scientific community. First, the theory has difficulties to predict the matter behaviour when the ion-ion coupling parameter $\Gamma_{i i}$ becomes larger than 1 , and second, it is difficult to realise experimental conditions that can create matter in such a state. The difficulty of irradiation conditions can be addressed with recent developments of the light source of $4^{\text {th }}$ generation. Indeed, XUV/X-ray Free Electron Lasers provide ultra short pulses of photons with short wavelengths at a high brightness, that is typically 10 order of magnitude higher than those of the previous generations. It provides sufficient energetic photons that can penetrate deeply into the matter to interact with a large number of atoms, which in turn emit enough radiations that can be recorded by spectroscopic techniques. Probing matter is possible by considering the X-ray self-emission from the target: these energetic transitions have a much larger mean free path compared to optical transitions and thus encounter a smaller number of absorption/re-emission process when escaping the matter at near solid density. Consequently, the analysis of the self X-ray emission is a probe of the matter under extreme conditions. We report here the $\operatorname{He}_{\alpha}$ emission of nitrogen $(\lambda=28.8 \AA)$

* Corresponding author: E-mail: frank.rosmej@upmc.fr 
originating from Boron Nitride (BN) target irradiated with an XUV-FEL radiation by using a large 2d dispersive Bragg crystal.

\section{Experimental setup}

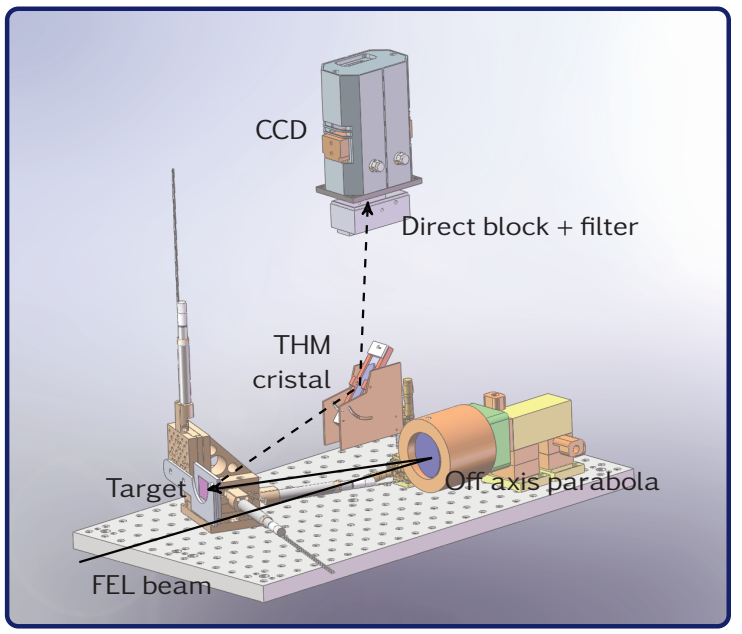

Fig. 1 Setup of the experimental chamber. The FEL beam comes from the right (solid line) and is tightly focused using the off-axis parabola coated for $92 \mathrm{eV}$ incoming photon energy. The thick target, when irradiated, emits X-ray photons (dashed line) of $0.43 \mathrm{keV}$ energy which are dispersed by a THM crystal up to a CCD on the top part of the scheme. A direct block and a $1200 \AA$ thick Carbon filter (LUXEL) are in front of the detector. (Color figure: www.cpp-journal.org).

In the framework of the Peek Brightness Collaboration, we used an experimental chamber designed for high vacuum, down to $10^{-6}$ torr. Figure 1 shows the path of the $92 \mathrm{eV}$ photons of the FLASH beam (solid line). They are focused using a Mo-Si coated multilayer off axis parabola with a focal length of $269 \mathrm{~mm}$. Observation of PMMA imprints revealed that the best focal spot size achieved was $0.8 \mu \mathrm{m}$ FWHM [1]. With about $10 \mu \mathrm{J}$ on target per pulse of $\sim 20 \mathrm{fs}$, at best focus we have reached an intensity larger than $10^{16} \mathrm{~W} / \mathrm{cm}^{2}$, providing unprecedent XUV-FEL irradiation conditions. The target was a solid $1 \mathrm{~mm}$ thick Boron Nitride foil. Here, Boron acts as a low Z substract for Nitrogen, the B K-shell emission does not overlap with X-ray emission from Nitrogen. The repetition rate of $5 \mathrm{~Hz}$ allowed us to accumulate 300 shots in a single CCD acquisition time. A step motor moved continuously the target in order to hit a fresh area at each shot. This was repeated 5 times, and then averaged in order to improve the signal to noise ratio. The spectrometer used a flat Tetra Hydrogen Maleate (THM) crystal with a $2 \mathrm{~d}=52.3 \AA$ allowing to disperse K-shell soft X-ray self-emission from BN target with a spectral resolution of about 300-400. The detector was an Andor CCD with pixel size of $26 \mu \mathrm{m} * 26 \mu \mathrm{m}$. The chip of the CCD was positioned inside the chamber and covered by a protective box blocking the direct radiation from the target and holding the filter (a $1200 \AA$ thick carbon film on a mesh, giving a transmission of about $59 \%$ for the $\mathrm{N} \mathrm{He}_{\alpha}$ at $28.8 \AA$ ). Spectra were integrated over time and space.

\section{K-shell emission from solid BN targets}

In order to characterize the interaction mechanism when irradiating solid BN targets $\left(\rho=2.18 \mathrm{~g} / \mathrm{cm}^{3}\right)$ with intense XUV-FEL, we have considered spectrally resolved $\mathrm{He}_{\alpha}$ line emission $(\sim 430 \mathrm{eV})$. Despite the fact that the spectrometer does integrate the signal over time, simulations indicate that the recorded emission allows to correlate the emission to times close to the laser pulse.

Figure 2 shows the experimental spectrum of the $\mathrm{N} \mathrm{He}_{\alpha}$-group emission which contains the $\mathrm{He}_{\alpha}$ resonance line emissions $1 \mathrm{~s} 2 \mathrm{p}{ }^{1} \mathrm{P}_{1}^{o}-1 \mathrm{~s}^{2}{ }^{1} \mathrm{~S}_{0}$, intercombination line $1 \mathrm{~s} 2 \mathrm{p}^{3} \mathrm{P}_{1}^{o}-1 \mathrm{~s}^{2}{ }^{1} \mathrm{~S}_{0}$ and adjacent dielectronic satellite emission $1 \mathrm{~s} 2 \operatorname{lnl}$ ' $-1 \mathrm{~s}^{2} \mathrm{nl}$ ' with $\mathrm{n}=2,3$, and 4 . The spectral location of these transitions is indicated in Figure 2 . This figure also includes results of simulations with the PrismSPECT code [2] that include non-LTE atomic population kinetics with ground, single and multiple excited states, as well as Stark broadening and radiative transport effects. The simulations reveal a significant density dependence (Figure 2) as well as a large temperature sensitivity (Figure 3). The best fit indicates that the emission occurs while the matter was heated up to about $90 \mathrm{eV}$ at still very high density, $\rho \sim 0.8 \mathrm{~g} / \mathrm{cm}^{3}$ which gives an electron density of about $3 \times 10^{22} \mathrm{~cm}^{-3}$. The soft X-ray emission occurs while the plasma drop, containing about $10^{10}$ particules with $10^{9}$ Helium-like nitrogen ions, was 
at a pressure of about $4 \times 10^{12} \mathrm{~Pa}$. Figure 2 shows that a rather good overall agreement with the observed data is obtained. The slight discrepancy near the short wavelengths side might be an artifact due to the geometrical end of the crystal/CCD.

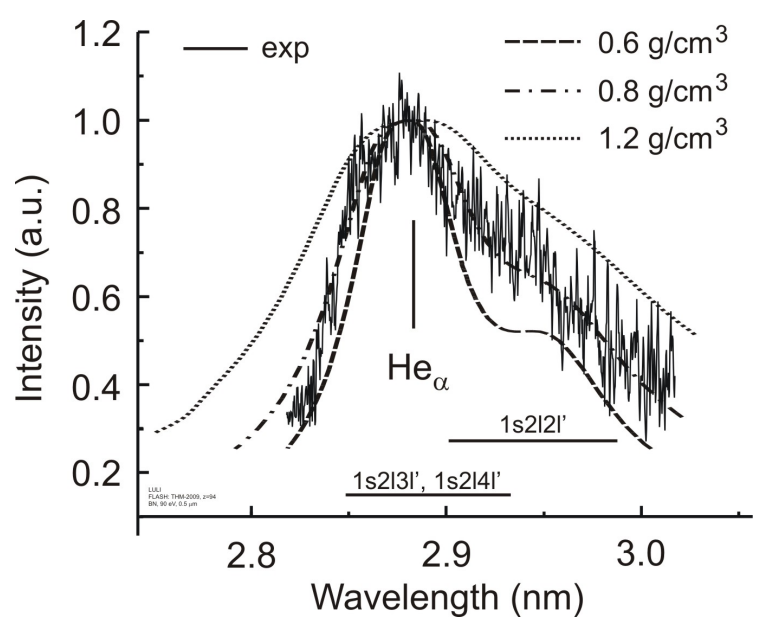

Fig. 2 K-shell emission spectrum of Nitrogen irradiating a solid $\mathrm{BN}$ target with the XUV-Free Electron Laser FLASH at $\lambda=13.5$ $\mathrm{nm}$ and laser intensities of $10^{16} \mathrm{~W} / \mathrm{cm}^{2}$. The simulations, which include radiation transport (source size $\mathrm{L}=0.5 \mu \mathrm{m}$ ) indicate that $\mathrm{He}_{\alpha}$ and its adjacent satellite transitions (indicated as 1s2121', $1 \mathrm{~s} 2131$ ' and $1 \mathrm{~s} 2141$ ') occur at near solid density $\rho \sim 0.8 \mathrm{~g} / \mathrm{cm}^{3}$.

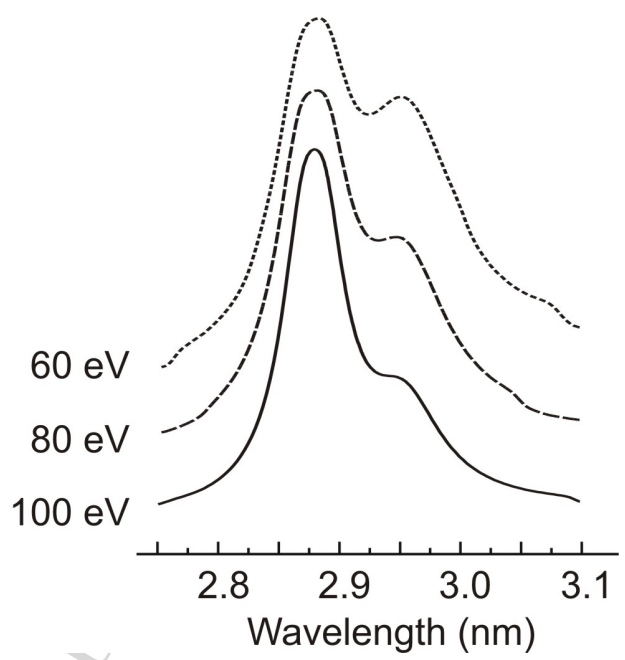

Fig. 3 Temperature sensitivity of $\mathrm{He}_{\alpha}$ and satellite transition of Nitrogen at near solid density : $\rho=0.6$ $\mathrm{g} / \mathrm{cm}^{3}, L=0.5 \mathrm{~mm}$ for electron temperature of 60 , 80 and $100 \mathrm{eV}$ (dotted, dashed and solid curve respectively). It shows that the observed line emission occurs at very high electron temperature, $\mathrm{kT}_{\mathrm{e}} \sim 90 \mathrm{eV}$.

The analysis allows to construct a picture of the electron heating when the XUV-FEL interacts with the solid BN. FEL photons with energy of $92 \mathrm{eV}$ allow to strip the 5 electrons of the L-shell [3], taking into account the possibility of 2s-intra-shell ionization. Since the $\mathrm{K}_{I}$-edge lies at $409.9 \mathrm{eV}$ [4], no other electron will be stripped by direct photoionization. Nitrogen is therefore left with 2 electrons in the K-shell after the pulse duration of $\sim 20$ fs.

Then, the thermalization of the photoionized free electrons starts, establishing an energy distribution function with rather high energy electrons in the tail in few fs [3]. For a Maxwellian energy distribution function with a temperature of $90 \mathrm{eV}$, about $1 \%$ of the free electrons have an energy larger than $430 \mathrm{eV}$, allowing collisional excitation of the $\mathrm{He}_{\alpha}$ resonance line emission and dielectronic capture into the 1s $21 n l$ '-configurations (which then gives rise to the dielectronic satellite structure indicated in Figure 2).

Simulations with the XRAD code [5] indicate that the main emission takes place in a time interval of about $200 \mathrm{fs}$ with the temperature between $80-110 \mathrm{eV}$ and densities between $0.4-2 \mathrm{~g} / \mathrm{cm}^{3}$. This is in excellent agreement with the spectral simulations (Fig. 2 and 3) which provided $\mathrm{kT}_{\mathrm{e}} \sim 90 \mathrm{eV}$ and $\rho \sim 0.8 \mathrm{~g} / \mathrm{cm}^{3}$.

The electron temperature of about $90 \mathrm{eV}$ indicates that a simple thermalization model of the photoionized electrons is insufficient. Indeed, the photoelectron energies are $(92 \mathrm{eV}-15 \mathrm{eV})=77 \mathrm{eV}$ for the $\mathrm{K}^{2} \mathrm{~L}^{5}$ configuration, $(92 \mathrm{eV}-29.5 \mathrm{eV})=62.5 \mathrm{eV}$ for the $\mathrm{K}^{2} \mathrm{~L}^{4}$ configuration and so on, which, after simple collisional thermalization, will lead to the temperature significantly smaller than $90 \mathrm{eV}$.

In conclusion, we have characterized the electron heating in XUV-FEL solid matter interaction experiments for the first time, employing K-shell emission spectroscopy. The deduced high electron temperature indicates that another heating mechanism, different from photoionization followed by Auger decay [6] has to be taken into account in order to explain the data resulting from the irradiation of solid Boron Nitride target by an intense XUV-FEL.

Acknowledgements The group from the Université Pierre et Marie Curie/LULI acknowledges the support of the Allianz Program of the Helmholtz Association, Contract No. EMMI HA-216 "Extremes of Density and Temperature: Cosmic Matter in the Laboratory". 


\section{References}

[1] J. Cihelka, L. Juha, J. Chalupský, F. Rosmej, O. Renner, K. Saksl, V. Hájková, L. Vyšín, E. Galtier, R. Schott, A. R. Khorsand, D. Riley, T. Dzelzainis, A. Nelson, R. Lee, P. Heimann, B. Nagler, S. Vinko, J.Wark, T.Whitcher, S. Toleikis, T. Tschentscher, R. Fäustlin, H. Wabnitz, S. Bajt, H. Chapman, J. Krzywinski, R. Sobierajski, D. Klinger, M. Jurek, J. Pelka, S. Hau-Riege, R.A. London, J. Kuba, N. Stojanovic, K. Sokolowski-Tinten, A. J. Gleeson, M. Störmer, J. Andreasson, J. Hajdu, and N. Timneanu, SPIE 7361, 73610P (2009).

[2] J.J. Mac Farlane et al., HEDP 3, 181 (2007).

[3] R.D. Cowan, The Theory of Atomic Structure and Spectra (University of California Press, Berkeley, 1980).

[4] B.L. Henke, E.M. Gullikson, and J.C. Davis, X-Ray Interactions: Photoabsorption, scattering, transmission, and reflection at $\mathrm{E}=5030000 \mathrm{eV}, \mathrm{Z}=1$ 92, Atomic Data Nucl. Data Tables, Vol. 54 (Elsevier, 1993).

[5] M. Fajardo, P. Zeitoun, and J.C. Gauthier, Eur. Phys. J. D 29, 69 (2004).

[6] E. Galtier, F. Rosmej, D. Riley, T. Dzelzainis, F. Khattak, P. Heimann, R. Lee, S. Vinko, T. Whitcher, B. Nagler, A. Nelson, J.Wark, T. Tschentscher, S. Toleikis, R. Fäustlin, R. Sobierajski, M. Jurek, L. Juha, J. Chalupsky, V. Hajkova, M. Kozlova, and J. Krzywinski, "XUV radiation emission originating from the solid-atomic transition phase induced by short intense free electron laser pulses", 2010, in preparation. 